\title{
Spin Polarized Transport through the Double-Dot System
}

\author{
D. SzTENKIEL AND R. ŚwiRKOWICZ
}

Faculty of Physics, Warsaw University of Technology

Koszykowa 75, 00-662 Warszawa, Poland

Spin-dependent electron transport through two quantum dots in series attached to ferromagnetic electrodes is analyzed within the framework of the non-equilibrium Green function formalism. Regime of a weak coupling between the dots is investigated. $I-V$ characteristics and tunnel magnetoresistance are calculated and discussed in detail.

PACS numbers: 75.20.Hr, 72.15.Qm, 72.25.-b, 73.23.Hk

\section{Introduction}

Spin-polarized transport in nanoscopic systems was mainly investigated for a single quantum dot (QD) attached to ferromagnetic electrodes (see e.g. [1]). It was shown that such a set could act as a spin valve. The current flowing through QD strongly depends on the configuration of magnetic moments in the leads. Usually, it is maximal for configuration in which magnetic moments are parallel in both electrodes. Spin-based devices are very important due to possibility of their application in spintronics. Especially, double quantum dot (DQD) structures could probably work in quantum computer hardware [2]. Electron transport through a double dot system received much experimental and theoretical attention during the past decade $[3,4]$, however, systems with non-magnetic electrodes were mainly investigated. Recently, spin-dependent transport has been studied for a set of two quantum dots capacitively coupled [5]. The linear conductance in the Kondo regime has been mainly investigated.

Here, we analyze spin-dependent transport through a system of two quantum dots in series. The inter-dot tunnel coupling can be easily tuned in such systems [6]. It strongly influences the current flowing through the system. Using the non-equilibrium Green function formalism based on the equation of motion we show that the DQD set can act as an effective spin valve in which not only tunnel magnetoresistance (TMR) value but also its sign can be easily changed with inter-dot coupling. 


\section{Model}

The system, which consists of two quantum dots in series attached to ferromagnetic electrodes, is described by the following Hamiltonian:

$$
H=H_{\mathrm{L}}+H_{\mathrm{R}}+H_{2 \mathrm{D}}+H_{\mathrm{T}} .
$$

The terms $H_{\beta}$ describe here the left $(\beta=\mathrm{L})$ and right $(\beta=\mathrm{R})$ electrodes in the non-interacting quasi-particle approximation $H_{\beta}=\sum_{k \sigma} \varepsilon_{k \beta \sigma} a_{k \beta \sigma}^{+} a_{k \beta \sigma}$ and $\varepsilon_{k \beta \sigma}$ is the single-electron energy in the $\beta$-th lead. The term $H_{2 \mathrm{D}}$ stands for DQD and is assumed in the form

$$
\begin{aligned}
H_{2 \mathrm{D}} & =\sum_{\beta \sigma}\left(E_{\beta} d_{\beta \sigma}^{+} d_{\beta \sigma}+\frac{1}{2} U_{\beta} d_{\beta \sigma}^{+} d_{\beta \sigma} d_{\beta-\sigma}^{+} d_{\beta-\sigma}\right) \\
& +V_{\mathrm{M}} \sum_{\sigma}\left(d_{\mathrm{L} \sigma}^{+} d_{\mathrm{R} \sigma}+\text { h.c. }\right) .
\end{aligned}
$$

$E_{\beta}$ denotes here the discrete level of the $\operatorname{dot} \beta(\beta=\mathrm{L}, \mathrm{R})$ and $U_{\beta}$ represents the intra-dot electron correlation parameter. Terms describing inter-dot Coulomb interactions are not taken into account in the Hamiltonian $H_{2 \mathrm{D}}$. The energy $E_{\beta}$ includes the electrostatic energy due to applied voltage $E_{\beta}=E_{\beta 0}+V_{\beta}^{\mathrm{d}}$, where $V_{\beta}^{\mathrm{d}}$ is the electrostatic potential of the $\beta$-th dot. The capacitance model allows us to compute the electrostatic potential fully self-consistently. The following formulae are used:

$$
\begin{aligned}
& e \sum_{\sigma}\left(n_{\mathrm{L} \sigma}-n_{\mathrm{L} \sigma}^{0}\right)=C_{\mathrm{L}}\left(V_{\mathrm{L}}-V_{\mathrm{L}}^{\mathrm{d}}\right)+C_{\mathrm{M}}\left(V_{\mathrm{L}}^{\mathrm{d}}-V_{\mathrm{R}}^{\mathrm{d}}\right), \\
& e \sum_{\sigma}\left(n_{\mathrm{R} \sigma}-n_{\mathrm{R} \sigma}^{0}\right)=C_{\mathrm{R}}\left(V_{\mathrm{R}}-V_{\mathrm{R}}^{\mathrm{d}}\right)+C_{\mathrm{M}}\left(V_{\mathrm{R}}^{\mathrm{d}}-V_{\mathrm{L}}^{\mathrm{d}}\right),
\end{aligned}
$$

in which $n_{\beta \sigma}$ and $n_{\beta \sigma}^{0}$ are occupation numbers of the $\operatorname{dot} \beta$ for a given and zero bias, respectively. $C_{\mathrm{L}}, C_{\mathrm{R}}, C_{\mathrm{M}}$ describe capacitances of the left, right, and inter-dot tunnel barriers. The last term in the Hamiltonian $H_{2 \mathrm{D}}$ (Eq. (2)) describes tunneling coupling between the two dots in series with the coupling parameter $V_{\mathrm{M}}$. Tunneling processes between DQD and external electrodes are included in $H_{\mathrm{T}}$ (Eq. (1)). Hamiltonian $H_{\mathrm{T}}$ takes the form $H_{\mathrm{T}}=\sum_{k \beta \sigma} t_{k \beta \sigma} a_{k \beta \sigma}^{+} d_{\beta \sigma}+$ h.c., where $t_{k \beta \sigma}$ denote elements of the tunneling matrix. In the following, we introduce parameters $\Gamma_{\beta \sigma}=2 \pi \sum_{k}\left|t_{k \beta \sigma}\right|^{2} \delta\left(E-\varepsilon_{k \beta \sigma}\right)$, which describe couplings between DQD region and external electrodes. $\Gamma_{\beta \sigma}$ are assumed to be constant within the electron band and to vanish outside the band. For systems with ferromagnetic electrodes $\Gamma_{\beta \sigma}$ depend on the spin index and polarization $p$ of the lead in the following way: $\Gamma_{\beta \sigma}=\Gamma_{\beta}(1+\hat{\sigma} p)$, where $\hat{\sigma}=1$ for electrons with majority spin and $\hat{\sigma}=-1$ for electrons with minority spin.

The non-equilibrium Green function formalism is introduced to describe electron transport in the non-linear regime. Green's functions are calculated in the Hartree-Fock (HF) approximation with the use of equation of motion (EOM) 
method. The procedure, which has been introduced to split higher order Green's functions, is justified for relatively small inter-dot coupling at temperatures higher than the Kondo temperature. The current flowing through the $\beta(\beta=\mathrm{L}, \mathrm{R})$ junction is calculated according to a standard formula [7]:

$$
I_{\beta}=\frac{\mathrm{i} e}{\hbar} \int \frac{\mathrm{d} E}{2 \pi} \sum_{\sigma} \Gamma_{\beta \sigma}\left[G_{\beta \sigma}^{<}+f_{\beta}\left(G_{\beta \sigma}^{\mathrm{r}}-G_{\beta \sigma}^{\mathrm{a}}\right)\right],
$$

where $f_{\beta}$ is the Fermi-Dirac distribution function in the lead $\beta$ and $G_{\beta \sigma}^{i}=$ $\left\langle\left\langle d_{\beta \sigma}, d_{\beta \sigma}^{+}\right\rangle\right\rangle^{i}$ denotes the Fourier transform of retarded, advanced, and lesser Green function for $i=\mathrm{r}$, a, $<$, respectively. On the basis of HF approximation the lesser Green function can be found from the appropriate EOM [8]. Taking into account the calculated formula for $G^{<}$one can express the current flowing in the biased system in the form

$$
I=\frac{e}{\hbar} \int \frac{\mathrm{d} E}{2 \pi} \sum_{\sigma}\left|G_{\mathrm{R} \sigma}^{0}\right|^{2}\left|G_{\mathrm{L} \sigma}\right|^{2} V_{\mathrm{M}}^{2} \Gamma_{\mathrm{L} \sigma} \Gamma_{\mathrm{R} \sigma}\left(f_{\mathrm{L}}-f_{\mathrm{R}}\right) .
$$

Due to relation $\left|G_{\beta \sigma}\right|^{2}\left|G_{-\beta \sigma}^{0}\right|^{2}=\left|G_{-\beta \sigma}\right|^{2}\left|G_{\beta \sigma}^{0}\right|^{2}$, which is fulfilled for the calculated Green functions, the current flowing through the system is conserved, $I_{\mathrm{L}}=-I_{\mathrm{R}}$ and the index $\beta$ was dropped. The mean occupation numbers $n_{\beta \sigma}=\left\langle d_{\beta \sigma}^{+} d_{\beta \sigma}\right\rangle$ on the $\operatorname{dot} \beta$ can be found according to the formula

$$
n_{\beta \sigma}=-\mathrm{i} \int \frac{\mathrm{d} E}{2 \pi} G_{\beta \sigma}^{<}=\int \frac{\mathrm{d} E}{2 \pi}\left(\Gamma_{\beta \sigma} f_{\beta}+\left|G_{-\beta \sigma}^{0}\right|^{2} V_{\mathrm{M}}^{2} \Gamma_{-\beta \sigma} f_{-\beta}\right)\left|G_{\beta \sigma}\right|^{2} .
$$

In the above expressions $G_{\beta \sigma}^{\mathrm{r}(\mathrm{a})}$ corresponds to retarded (advanced) Green function of the dot $\beta$ calculated from EOM and $\left|G_{\beta \sigma}^{0}\right|^{2}=\left(A_{\beta \sigma}^{2}+\frac{1}{4} \Gamma_{\beta \sigma}^{2}\right)^{-1}$ with

$$
A_{\beta \sigma}=\frac{\left(E-E_{\beta}\right)\left(E-E_{\beta}-U_{\beta}\right)}{E-E_{\beta}-U_{\beta}\left(1-n_{\beta-\sigma}\right)} .
$$

Current flowing through the system and occupation numbers are calculated in a self-consistent way.

\section{Numerical results}

Electric current flowing through DQD system and number of electrons are calculated numerically with the use of parameters: $E_{\mathrm{L}}=E_{\mathrm{R}}=E_{0}=4, U_{\mathrm{L}}=$ $U_{\mathrm{R}}=U=8, \Gamma_{\mathrm{L}}=\Gamma_{\mathrm{R}}=\Gamma=1, C_{\mathrm{L}}=C_{\mathrm{R}}=C_{\mathrm{M}}=4, k T=1$. It is assumed that energy levels in both dots are aligned for zero bias. With increasing voltage the dot levels start to split. $I-V$ characteristics calculated for DQD attached to non-magnetic leads $(p=0)$ are very similar to the ones found by Niu and Liu [9]. Here, we report only results obtained for systems with ferromagnetic electrodes characterized by polarization factor $p$. $I-V$ characteristics were taken for various values of inter-dot coupling $V_{\mathrm{M}}$ and polarization factor $p$. Two collinear magnetic configurations, parallel $(\mathrm{P})$ and antiparallel (AP) of magnetic moments 
in the electrodes were investigated. Current flowing through the system in $\mathrm{P}$ configuration depends very weakly on the polarization factor. Typical curves are presented in Fig. 1a. As a function of bias voltage the current shows two well visible peaks. The first one appears when the level $E_{0}$ enters a tunneling window, whereas the second peak corresponds to $E_{0}+U$. Intensities of the peaks strongly depend on the coupling parameter $V_{\mathrm{M}}$ and increase with increasing $V_{\mathrm{M}}$. For the case of two identical dots, investigated here, the current is fully symmetric for the bias reversal. In $\mathrm{P}$ configuration numbers of electrons accumulated on $\mathrm{L}$ and $\mathrm{R}$ dots do not strongly depend on the coupling parameter $V_{\mathrm{M}}$ (Fig. 1b). For negative voltages (positive $\mathrm{eV}$ according to the figure) number of electrons starts to increase on the left dot. For higher bias the L dot is practically doubly occupied, whereas
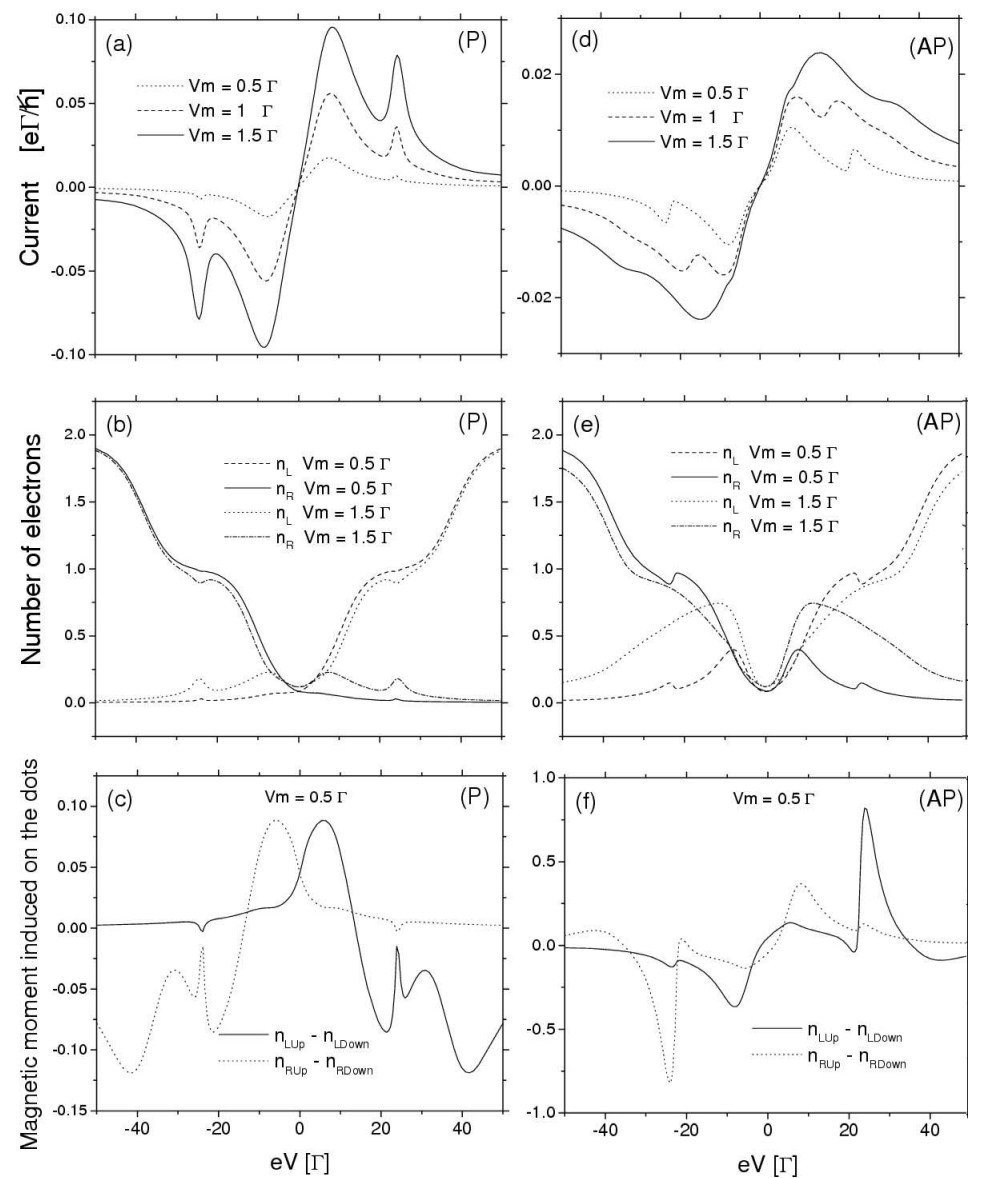

Fig. 1. Bias dependence of the current (a,d), occupation numbers (b,e) and magnetic moment induced on the dots (c,f) calculated for parallel (left part) and antiparallel (right part) configurations of magnetic moments in the electrodes with polarization factor $p=0.95$. 
the right one is practically empty. For positive voltages $(e V<0)$ the situation is reversed. Electrons which occupy the dots are practically spin-degenerated and only small magnetic moment is induced on each dot for highly polarized external electrodes (see Fig. 1c). Quite different situation takes place in AP configuration. In this case the current strongly depends on polarization factor of the leads. For small $p$ factor current flowing in the system does not differ essentially from the one typical of $\mathrm{P}$ configuration. However, for large $p$ it changes dramatically. Current intensity lowers substantially with an increase in $p$. Moreover, two-peak structure is now not very well visible. For high values of $p$ and relatively strong inter-dot coupling the current shows round and small cusp (Fig. 1d). When electrodes are strongly polarized $(p=0.95)$ both dots are occupied with electrons in the small bias region. Quite large magnetic moment is induced. But, for high bias voltages one dot is practically doubly occupied and the other is empty. The moment tends to zero (Figs. 1e,f).

The difference between currents flowing in $\mathrm{P}$ and $\mathrm{AP}$ configurations gives contribution to tunnel magnetoresistance, which can be determined as

$$
\mathrm{TMR}=\frac{I^{\mathrm{P}}-I^{\mathrm{AP}}}{I^{\mathrm{AP}}},
$$

where $I^{\mathrm{P}}\left(I^{\mathrm{AP}}\right)$ denotes the current in $\mathrm{P}(\mathrm{AP})$ configuration. Figure 2 presents
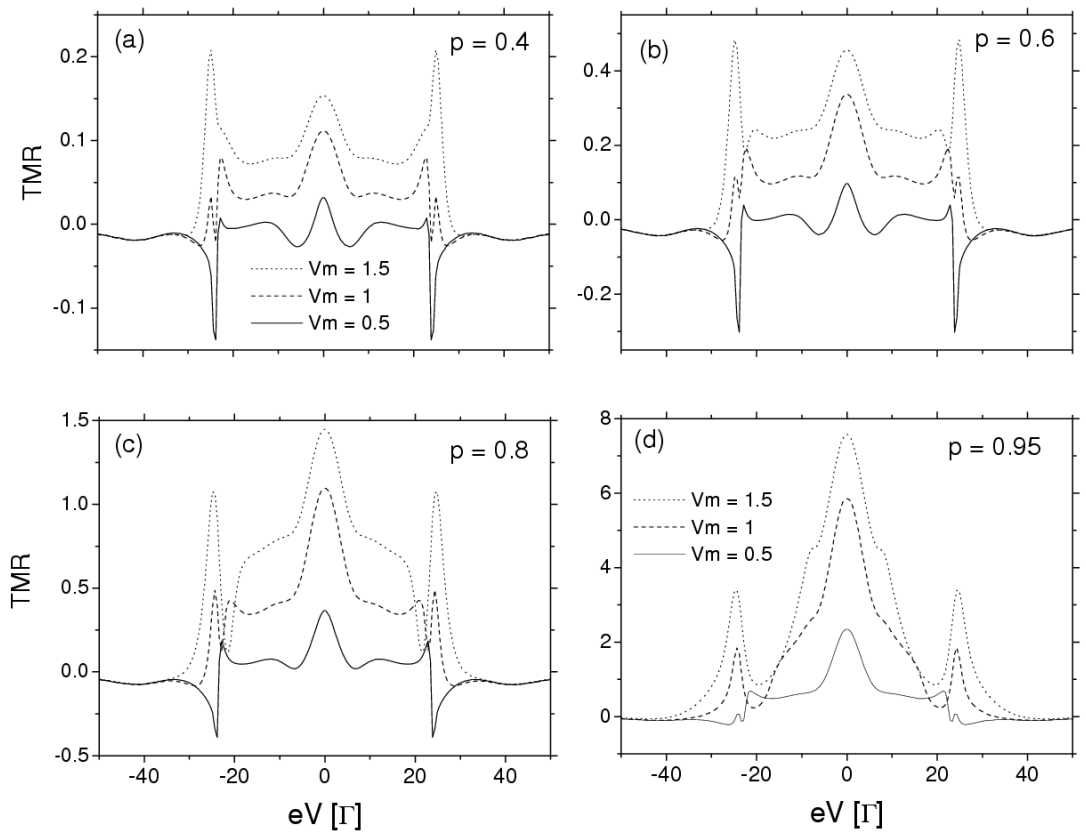

Fig. 2. Bias dependence of TMR calculated for indicated values of polarization factor (a-d) and inter-dot coupling parameter. 
TMR in dependence on bias voltage for different polarization factors. In the Coulomb blockade region (small voltages) TMR is enhanced and positive. For voltages corresponding to the second peak in the current TMR can be negative. This change of sign takes place only for a weak inter-dot coupling $\left(V_{\mathrm{M}}=0.5\right)$. When $V_{\mathrm{M}}$ increases, TMR strongly increases and takes positive values in the whole bias voltage region. TMR also strongly increases with the polarization factor. For high values of $p(p=0.95)$ TMR is considerably enhanced. One can see that in systems with two quantum dots in series attached to ferromagnetic electrodes TMR can be easily changed with inter-dot coupling. For strong $V_{\mathrm{M}}$ the curves start to represent behavior typical of TMR in one-dot system [10].

\section{References}

[1] J. Barnaś, A. Fert, Phys. Rev. Lett. 80, 1058 (1998).

[2] D. Loss, D.P. DiVincenzo, Phys. Rev. B 57, 120 (1998).

[3] K.A. Matveev, L.I. Glazman, H.U. Baranger, Phys. Rev. B 54, 5637 (1996).

[4] W.G. Van der Wiel, Rev. Mod. Phys. 75, 1 (2003).

[5] B. Bułka, S. Lipiński, Nonlinear Optics, Quantum Optics 30, 191 (2003).

[6] L.P. Kouwenhoven, C.M. Marcus, in: Mesoscopic Electron Transport, Eds. L.L. Sohn, L.P. Kouwenhoven, G. Schön, Kluwer, Dordrecht 1997, p. 105.

[7] H. Haug, A.P. Jauho, Quantum Kinetics in Transport and Optics of Semiconductors, Springer Verlag, Berlin 1996.

[8] C. Niu, D.L. Lin, T.H. Lin, J. Phys., Condens. Matter 11, 1511 (1999).

[9] C. Niu, L. Liu, Phys. Rev. B 51, 5130 (1995).

[10] W. Rudziński, J. Barnaś, R. Świrkowicz, M. Wilczyński, Phys. Rev. B 71, 205307 (2005). 